\title{
Neuroimaging and Fetal Alcohol Spectrum Disorders
}

\author{
Andrea D. Spadoni ${ }^{1}{ }^{*}$, Christie L. McGee ${ }^{1}$, Susanna L. Fryer ${ }^{1}$, and Edward P. Riley ${ }^{2}$ \\ 1 Joint Doctoral Program in Clinical Psychology, San Diego State University / University of California, San \\ Diego \\ 2 Department of Psychology and the Center for Behavioral Teratology, San Diego State University, San \\ Diego, CA, USA
}

\begin{abstract}
Heavy prenatal alcohol exposure causes permanent structural alterations to the brain and can lead to numerous cognitive and behavioral outcomes. Consistent with many of the neuropsychological and behavioral deficits that have been reported, neuroimaging studies reveal a pattern of structural abnormalities associated with prenatal alcohol exposure. This chapter systematically reviews structural anomalies by brain region, identifying cognitive and behavioral correlates when relevant. The consensus shows that in addition to the overall reduction of brain size, prominent brain shape abnormalities have been observed, with narrowing in the parietal region and reduced brain growth in portions of the frontal lobe. Commensurate with these anomalies, volumetric and tissue density findings cite disproportionate reductions in the parietal lobe, cerebellar vermis, corpus callosum, and the caudate nucleus, suggesting that certain areas of the brain may be especially vulnerable to prenatal alcohol exposure. In sum, neuroimaging techniques have greatly advanced our understanding of brain-behavior relationships in fetal alcohol spectrum disorders (FASD), and hopefully will lead to improved diagnosis and treatment options for those affected by prenatal exposure to alcohol.
\end{abstract}

\section{Keywords}

fetal alcohol syndrome; prenatal alcohol exposure; fetal alcohol spectrum disorders; teratogenesis; brain imaging; basal ganglia; corpus callosum; cerebellum; magnetic resonance imaging

\section{Introduction}

The devastating effects of prenatal alcohol exposure on the developing embryo and fetus represent a continuum of physical anomalies and behavioral neurocognitive deficits that may affect as many as one percent of all births (May and Gossage, 2001; Sampson et al., 1997). As Jones and colleagues termed it in 1973, fetal alcohol syndrome (FAS) denotes a triad of characteristics: (1) evidence of central nervous system (CNS) dysfunction, (2) craniofacial anomalies, e.g. indistinct philtrum (groove between nose and upper lip), thin upper vermillion border (lip) and small palpebral fissures (eye openings), and (3) pre- and/or post-natal growth deficiencies (Jones and Smith, 1973; Jones et al., 1973). More recently "fetal alcohol spectrum disorders," or FASD, has come into usage as a nondiagnostic, umbrella term used to describe

\footnotetext{
*Corresponding author: Edward Riley, Center for Behavioral Teratology 6363 Alvarado Ct. \#209, San Diego, CA 92120, USA. Phone: 619-594-4601. Fax: 619-594-1895.

This work was supported by grants R01AA010417 and T32AA013525 from NIAAA.

Publisher's Disclaimer: This is a PDF file of an unedited manuscript that has been accepted for publication. As a service to our customers we are providing this early version of the manuscript. The manuscript will undergo copyediting, typesetting, and review of the resulting proof before it is published in its final citable form. Please note that during the production process errors may be discovered which could affect the content, and all legal disclaimers that apply to the journal pertain.
} 
the entire range of effects resulting from gestational alcohol exposure (http://www.nofas.org/healthcare/indicators.aspx). Non-dysmorphic FASD individuals, though lacking the facial criteria necessary for an FAS diagnosis, often have well-documented functional and behavioral deficits that persist into adulthood (Olson et al., 1998; Jacobson et. al., 1994; Mattson et al., 1998; Roebuck et al., 1998a; Streissguth et al., 1994, 1995). In addition to cognitive and behavioral consequences, FASD also pose an economic burden. The cost of dealing with the consequences of prenatal alcohol exposure is estimated at $\$ 4$ billion dollars per year in the United States (Harwood, 2000). Clearly, these public health and economic concerns warrant continued research to develop systematic identification, effective interventions, and improved outcomes for individuals with FASD. The application of brain imaging methods such as magnetic resonance imaging (MRI), has allowed us to better understand the neural bases underlying the behavioral problems frequently reported following gestational alcohol exposure. Research based on neuroimaging techniques may improve diagnostic possibilities, which will hopefully lead to an improved future for those affected by prenatal alcohol exposure.

\section{Autopsy and the Advantages of Imaging Techniques}

Prior to the implementation of neuroimaging techniques, researchers relied upon autopsy cases to characterize brain morphology in FAS. A wide range of neuropathological findings were reported, including: hydrancephaly, anencephaly, holoprosencephaly, cerebral dysgenesis, abnormal neural migration, hydrocephaly, microcephaly/microencephaly, enlarged/reduced/ absent ventricles, and severe CNS disorganization. Furthermore, abnormalities of the basal ganglia, diencephalon, cerebellum, brainstem, optic nerve, olfactory bulb, hippocampus, pituitary, and neural tube were reported (Clarren, 1986; Clarren et al., 1978; Coulter et al., 1993; Jones and Smith, 1975; Jones et al, 1973; Peiffer et al., 1979; Wisniewski et al., 1983). Such heterogeneous brain malformations led to the conclusion that no identifiable pattern of brain abnormalities specific to FAS existed (Clarren et al., 1978; Peiffer et al., 1979).

However, evidence gathered postmortem does not necessarily represent the brain changes seen in those living with FAS. Although evidence obtained via autopsy in individuals with FAS was useful, in many cases it described brain abnormalities incompatible with life. Neuroimaging methods, as opposed to autopsy studies, have the obvious advantage of characterizing the effects of prenatal alcohol on the living brain, providing the opportunity to study a more generalizable sample of the FASD population. In contrast to the diverse autopsy findings, imaging studies comparing subjects with FASD to typically developing controls have identified patterns of sparing and loss associated with prenatal alcohol exposure. This chapter reviews FASD-focused neuroimaging research. Structural anomalies will be discussed by brain region, and cognitive and behavioral correlates of structural abnormalities will be noted when relevant.

\section{Cerebral Volume and Shape}

Diagnostic guidelines for FAS include microcephaly as one indicator of a CNS deficit. As might be expected, MRI studies routinely report analogous results citing overall reductions in the cranial, cerebral, and cerebellar vaults in FAS (Archibald et al., 2001; Autti-Ramo et al., 2002; Mattson et al., 1992, 1994, 1996; Sowell et al., 2001a, 2002a; Swayze et al., 1997). Volume Analyses.

To determine if specific lobes of the cerebral volume were more affected than other, Archibald et al, (2001) used traditional segmentation and parcellation methods and reported hypoplasia in the parietal, temporal, and frontal lobes of children and adolescents with FAS compared to normal developing controls (Archibald et al., 2001). However, once overall brain volume was accounted for, only reductions in the parietal region remained statistically significant. The 
nondysmorphic alcohol exposed group did not differ from the controls on any of the volume measures.

To further explore areas of the brain that may be selectively vulnerable to alcohol exposure, voxel-based morphometric (VBM) analyses were used to evaluate tissue density of the brains of adolescents prenatally exposed (Sowell et al., 2001a). This novel technique compares signal intensity across voxels, or cubic pixels, of a brain image. Signal intensity is then used as a proxy for tissue density. VBM based findings were concordant with studies using more traditional techniques and demonstrated greater regional hypoplasia in posterior temporoparietal lobes than in temporal and occipital lobes in the brains of adolescent exposed to alcohol prenatally (Sowell et al., 2001a). After controlling for overall brain size, patterns of decreased volume in the parietal regions remained significant. In this study the nondysmorphic FASD group and FAS group were combined when compared to controls. In a subsequent study, (Sowell et al., 2002a), volumetric analysis indicated that the parietal lobes were the most affected, though volume reductions were also noted in the frontal and temporal lobes as well. Again after correcting for overall brain volume reductions, only the parietal lobe volumes were significantly different than controls.

\section{Regional Brain Shape Abnormalities}

In order to assess regional shape, Sowell and colleagues (2002a) employed whole-brain surface analyses. By identifying sulcal and gyral markers and comparing "the distance from the center" (DFC), or the measurement from a particular point on the anterior commissure to these landmarks, relationships between brain shape and local matter densities were observed. In participants exposed to alcohol prenatally the DFC in bilateral inferior parietal regions was reduced, giving these regions a "narrow" appearance as compared to controls. Furthermore, measurements from the "center of the brain" to landmarks on the cortical surface of the left orbito-frontal corticies were shorter in subjects with FASD than in controls, creating a "blunted" effect in the frontal lobe.

\section{Gray and White Matter Differences}

In their 2001 paper, Archibald et al. reported a proportional reduction in the amount of white matter in the cerebrum. A smaller proportional reduction of white matter in the cerebellum did not reach significance. They concluded that white matter hypoplasia might be more severe than gray matter changes in FAS. Subsequently, Sowell et al. (2002a) found that structural irregularities also corresponded to areas of increased gray matter density and/or white matter hypoplasia (Sowell et al., 2002a). Density maps show gray matter as significantly increased in bilateral (and especially left hemispheric) inferior parietal regions, where alcohol-exposed participants had shortened DFC measures. Thus, at least in the parietal lobe, decreased lobar volume appears to occur in the context of increased gray matter and decreased white matter densities.

Based on the findings of left hemispheric growth reductions, Sowell and colleagues (2002b) examined hemispheric asymmetry patterns in a sample of alcohol-exposed adolescents. Cortical matching techniques revealed that individuals with FAS and FASD had significantly reduced "right to left" gray matter ratios in the posterior temporal lobes, compared to typically developing adolescents. The authors suggest that these findings may relate to deficits that individuals with prenatal alcohol exhibit in language processing and object recognition.

In conclusion, the results of these various analyses suggest that individuals exposed to heavy amounts of alcohol prenatally exhibit regional growth deficiencies in the parietal and perhaps orbito-frontal regions, beyond overall reductions of total brain volume. Furthermore, white matter hypoplasia found throughout the brains of alcohol exposed individuals was most 
prevalent in the perisylvian region and inferior parietal regions. Additionally, gray matter increases in density were noted in inferior parietal regions and superior temporal lobes, suggesting an inverse relationship between regional size and gray matter density. Finally, frontal and parietal lobe anomalies are consistent with executive functioning deficits shown in individuals with FASD such as poor planning, cognitive inflexibility, and reduced response inhibition and with spatial memory problems. (Kodituwakku et al., 2001; Mattson et. al., 1999). The parietal lobe deficits might

\section{Cerebellum}

The cerebellum is associated with motor functions such as posture, balance, and coordination. Regions of the cerebellum are also thought to contribute to attention (Devinsky and D'Esposito, 2004) and classical conditioning (Kim and Thompson, 1997).

A finding commonly ascribed to prenatal alcohol exposure is reduced surface area (Autti-Ramo et al., 2002) and volume of the cerebellum (Mattson et al., 1992; Archibald et al., 2001), suggesting yet another brain area particularly sensitive to alcohol exposure. In addition to reduced cerebellar volume, studies report damage localized to particular regions of the cerebellum (Autti-Ramo et al., 2002; O'Hare et al., 2005; Sowell et al., 1996). In both nondysmorphic alcohol-exposed and FAS subjects, volume in the anterior cerebellar vermis (lobules I-V), an earlier developing part of the cerebellum, was significantly reduced after controlling for reduced brain size (Autti-Ramo et al., 2002; Sowell et al., 1996) while later developing regions were relatively spared (Sowell et al., 1996; Autti-Ramo et al., 2002; O'Hare et al., 2005). Analyses also revealed that the superior and anterior edges of the anterior vermis appear to be displaced in alcohol-exposed subjects as compared to controls (O'Hare et al., 2005). Participants with a diagnosis of FAS showed the greatest amount of displacement (1$3 \mathrm{~mm}$ ), while non-dysmorphic individuals with alcohol exposure histories showed less dramatic $(1.4 \mathrm{~mm})$ alterations (O'Hare et al., 2005).

Given the nature of functions ascribed to the cerebellum, structural anomalies may contribute to the functional deficits commonly observed in FASD, such as in balance, bimanual coordination (Roebuck et al., 1998b; Roebuck-Spencer et al., 2004), and attention (Coles et al., 1997, 2002; Lee et al., 2004; Streissguth et al., 1995). In addition, vermal dysmorphology has been shown to be negatively correlated with verbal learning and memory in an alcoholexposed group (O'Hare et al., 2005). Therefore, understanding alcohol-induced damage to the cerebellum may play an important role in seeking an identifiable pattern of neurocognitive deficits related to FASD.

\section{Corpus Callosum}

The corpus callosum (CC) is a white matter tract connecting the two cerebral hemispheres. Because it is the major conduit for cerebral inter-hemispheric transfer, the CC plays a role in the coordination of various functions such as bimanual motor tasks, sustained attention, vision, as well as spatial and visual working memory (Devinsky and D’Esposito, 2004).

Early autopsy studies on FAS cases (Jones et al., 1973; Clarren et al., 1978; Peiffer et al., 1979; Wiseniewski et al., 1983) reported agenesis, or absence, of the corpus callosum (ACC). Although total absence of this brain structure is rare, occurring at a rate of $0.1 \%$ in the typical population and $2 \%$ in the developmentally disabled (Jeret et al., 1986), a higher rate of ACC has been reported in individuals with FAS (Riley, et al, 1995; Swayze et al. 1997). Also, ACC is commonly accompanied by ventricular abnormalities, as midline structures typically adjoining the corpus callosum are displaced (Johnson et al., 1996). 
While ACC is rare even among individuals with FAS, hypoplasia of the corpus callosum has been frequently reported (Autti-Ramo et al., 2002; Mattson et al., 1992; Riley et al., 1995; Sowell et al. 2001b; Swayze et al., 1997). In these studies, midsagittal MRI images were used to systematically measure structural integrity of the corpus callosum. A large San Diego based study found that after controlling for reduced overall brain size, the FASD group had significant reductions in anterior and posterior callosal regions (Riley et al., 1995). A study employing similar methodology, using a subsample of the San Diego study, also found that the posterior section (splenium) of the corpus callosum was significantly reduced in area as well as significantly displaced in the FASD group relative to control participants (Sowell et al., 2001b). Among the nondysmorphic FASD cases, this inferior and anterior displacement of the splenium occurred to a lesser degree than in that of cases with FAS. Thus, these regional abnormalities may suggest that sections of the corpus callosum are differentially sensitive to prenatal alcohol exposure.

Rather than compare area, volume, or location, other studies have described the shape of the corpus callosum in FAS and other FASD individuals (Bookstein et al., 2001, 2002a). Using novel shape-based representations of callosal morphology, alcohol-exposed subjects exhibited increased variability in the shape of the CC (Bookstein et al., 2001, 2002a). According to Bookstein et al. (2001), the shape-based analytic technique may be more valid than previously used techniques as it requires no spatial correction for microcephaly commonly found in alcohol-exposed groups. Additionally, shape-based analyses of the corpus callosum may provide an additional means of identifying individuals with FASD, as this technique accurately classified group membership in 100 of 117 (85\%) alcohol exposed subjects (Bookstein et al., 2002a). Therefore, shape-based analysis may prove an advance for the diagnosis of FAS.

Recently, diffusion tensor imaging (DTI) was used to evaluate the white matter integrity of the corpus callosum in alcohol-exposed participants (Ma et al., 2005). Diffusion tensor imaging (DTI) makes use of the random properties of water diffusion to generate images that can be used to evaluate tissue microstructure. Two important variables of interest in DTI are the apparent diffusion coefficient (ADC) and fractional anisotropy (FA). ADC is a measure of the ability of water to diffuse within a given tissue compartment, averaged across spatial directions. FA is an index measure of anisotropy, where values range from 0 to 1 , with higher values indicating more restricted diffusion, and thus more coherent microstruture.

In a small study of young adults, alcohol-exposed individuals had decreased FA and increased $\mathrm{ADC}$ in the genu and splenium of the corpus callosum as compared to controls, which is suggestive of less coherent white matter formation (Ma et al., 2005). However, FA and ADC were not found to be related to any specific neurocognitive deficits. Finally, it is important to note that although the groups were statistically different on measures of FA and ADC, the alcohol-exposed sample did not show statistically reduced intercranial volumes or hypoplasia of the corpus callosum although there was a statistical trend towards a reduction similar to previous findings. While CC hypoplasia has been consistently associated with prenatal alcohol exposure, (Archibald et al, 2001; Bhatara et al., 2002; Riley et al., 1995; Sowell et al., 2001b), it may be that white matter microstructure is a more sensitive index of alcohol-exposure than these macrostructural markers (Ma et al., 2005). In summary, evidence from MRI suggests that like the cerebellum, the corpus callosum may show increased vulnerability to gestational alcohol exposure.

Many studies suggest a relation between functional deficits and callosal dysmorphology, especially in bimanual coordination (Roebuck-Spencer et al. 2004), attention (Coles et al., 1997, 2002), executive functioning (Bookstein et al., 2002b; Kodituwakku et al., 2001; Mattson et al., 1999), and verbal learning abilities (Sowell et al., 2001b). There is also evidence that certain anomalies are related to specific cognitive deficits. For example, studies on callosal 
location found that greater anterior displacement of the splenium and isthmus predicted poorer verbal learning ability (Sowell et al., 2001b). In addition, "thick" corpora callosa (blunted in the anterior portion and thereby projecting less into the frontal regions) have been correlated with poorer executive functioning, suggesting that white matter tracts connecting brain areas involved in executive functions in the anterior portions of the corpus callosum may be compromised (Bookstein et al., 2002b). Additionally, "thin" corpora callosa have been related to motor deficits (Bookstein et al., 2002b). In this case, connections within the CC may be less efficient in delivering information to the brain's motor centers. These results are also concordant with reports of decreased performance on spatial and visual memory tasks in FASD (Mattson \& Riley, 1998; Streissguth et. al., 1994). Taken together, neuropsychological correlates of callosal anomalies suggest increased shape variability, displacement, and reduced area are associated with decreased performance in a wide range of functional and cognitive arenas in individuals prenatally exposed to alcohol.

\section{Basal Ganglia}

The basal ganglia are composed of five principal nuclei: the caudate nucleus (cognitive striatum), nucleus accumbens (limbic striatum), putamen (sensorimotor striatum), globus pallidus (GP) and subthalamic nucleus (Devinsky and D'Esposito, 2004). Together, the putamen and globus pallidus are often referred to as the lenticular nucleus. The basal ganglia reside deep inside the brain, and are heavily connected to cortical and subcortical motor areas and are thus intimately related to movement and procedural learning. In addition, the basal ganglia are involved in cognitive, affective, and motivational functions (Devinsky and D’Esposito, 2004).

Evidence of reduced volume of the basal ganglia in prenatally alcohol exposed individuals has been widely reported (Archibald et al., 2001; Mattson et al., 1992, 1994, 1996). In addition, gray matter has been shown to be disproportionately affected (Mattson et al., 1992). However, these regional abnormalities in the basal ganglia remain evident only in the caudate nucleus after controlling for an overall decrease of volume in the cerebral vault (Archibald et al., 2001; Mattson 1992, 1994, 1996), while the volume of the lenticular nucleus and nucleus accumbens remained relatively spared (Archibald et al., 2001; Mattson et al., 1996). Additionally, this pattern of abnormalities is thought to be closely related to many of the functional deficits seen in FASD individuals, for instance, deficits in spatial memory, perseveration, and difficulty set shifting. Thalamic abnormalities may also be related to deficits in motor coordination reported in exposed individuals (Clark et al., 2000, Mattson and Riley, 1998). Researchers also propose that deficits in executive functioning, spatial learning, attention, perseverative behavior, and response inhibition may be related consequences (Mattson et al., 1996) due to the hypothesized association between executive functions and frontal-striatal connectivity. Therefore, specific regional abnormalities in the basal ganglia are thought to moderate many of the functional difficulties experienced by FASD individuals.

\section{Hippocampus and Amygdala}

The hippocampus and amygdala belong to the subcortical limbic system which mediates affective behavior. The hippocampus is an essential component of the human memory network and is integral to the synthesis of learned information as well as information recovery during retrieval (Devinsky and D'Esposito, 2004). Studies also show that the hippocampus may be involved in spatial learning abilities (McClelland and Goddard, 1996). The amygdala processes emotional input and relays it to neocortical limbic, sensory, and cognitive regions. In addition, regulation of emotion and affective expression are related to this structure (Devinsky and D’Esposito, 2004). 
Imaging data provides conflicting evidence as to whether a specific pattern of regional abnormalities exist in the hippocampi of individuals with FASD. Reports of hippocampal abnormalities have been isolated to a few individuals rather than displaying a consistent withingroup pattern of alteration (Autti-Ramo et al., 2002; Bhatara et al., 2002). In fact, some studies have reported relative sparing of the hippocampus and amygdala (Archibald et al., 2001; Mattson et al., 1994). However, a small study reported asymmetrical hippocampal formations in the FASD group, citing right larger than left hippocampal volumes (Riikonen et. al., 1999). Because deficits in place learning have been reported in alcohol exposed individuals, further clarification of this structure and the effects of gestational alcohol exposure are warranted (Hamilton et al., 2003).

\section{Summary and Conclusions}

Brain imaging techniques have shown that prenatal exposure to alcohol causes permanent structural alterations to the brain. Studies have demonstrated reduced overall volume of the FASD brain. Moreover, certain areas of the brain may be more vulnerable to exposure, as studies have demonstrated damage to the corpus callosum, cerebellar vermis, basal ganglia, as well as perislyvian, orbito-frontal, and parietal brain regions of FASD individuals. In contrast to the heterogeneous conclusions from autopsy studies, these imaging results indicate that a specific pattern of change in the wake of gestational alcohol exposure exists.

Neuropsychological theory indicates that the functional and behavioral sequelae of FASD may directly correspond to these insults, as deficits in behavioral inhibition, executive functioning, learning, memory, and attention have been found to characterize samples of alcohol-exposed individuals.

Finally, the goal of this review was to demonstrate that neuroimaging techniques have advanced our understanding of brain-behavior relationships in FASD. Future research is necessary to independently confirm these results, as well as improve our current understanding of how gestational alcohol exposure impacts the brain. Continuing to develop and support novel methodologies is crucial in order to validate and augment our existing knowledge. Functional (fMRI) and magnetic resonance spectroscopy (MRS) are imaging techniques that hold promise for advancing the study of FASD. MRS can be used to detect chemical elements, such as markers of neuronal integrity and cell death (Adalsteinsson et al., 2002). fMRI provides a proxy of brain activity and may help characterize the neural underpinnings of abnormalities observed among individuals affected by FASD while engaged in some behavior and help develop and target interventions. By focusing on neuropsychological correlates to structural, chemical, and functional alterations due to prenatal alcohol exposure future studies will advance our understanding of the behavioral and neurocognitive phenotype of FASD, as well as our current brain-behavior assumptions.

\section{References}

Adalsteinsson, E.; Sullivan, EV.; Pfefferbaum, A. Biochemical, functional and microstructural magnetic resonance imaging (MRI). In: Liu, Y.; Lovinger, DM., editors. Methods in Alcohol-Related Neuroscience Research. CRC Press; Boca Raton: 2002. p. 345-371.

Archibald SL, Fennema-Notestine C, Gamst A, Riley EP, Mattson SN, Jernigan TL. Brain dysmorphology in individuals with severe prenatal alcohol exposure. Dev Med Child Neurol 2001;43:148-154. [PubMed: 11263683]

Autti-Ramo I, Autti T, Korkman M, Kettunen S, Salonen O, Valanne L. MRI findings in children with school problems who had been exposed prenatally to alcohol. Dev Med Child Neurol 2002;44:98106. [PubMed: 11848116]

Bhatara VS, Lovrein F, Kirkeby J, Swayze V II, Unruh E, Johnson V. Brain function in fetal alcohol syndrome assessed by single photon emission computed tomography. S D J Med 2002;55:59-62. [PubMed: 11865707] 
Bookstein FL, Sampson PD, Streissguth AP, Connor PD. Geometric morphometrics of corpus callosum and subcortical structures in the fetal-alcohol affected brain. Teratol 2001;64:4-32.

Bookstein FL, Sampson PD, Connor PD, Streissguth AP. Midline corpus callosum is a neuroanatomical focus of fetal alcohol damage. Anat Rec 2002a;269:162-174. [PubMed: 12124903]

Bookstein FL, Streissguth AP, Sampson PD, Connor PD, Barr HM. Corpus callosum shape and neuropsychological deficits in adult males with heavy fetal alcohol exposure. NeuroImage 2002b; 15:233-251. [PubMed: 11771992]

Clark CM, Li D, Conry J, Conry R, Loock C. Structural and functional brain integrity of fetal alcohol syndrome in nonretarded cases. Pediatrics 2000;105:1096-1099. [PubMed: 10790468]

Clarren, SK. Neuropathology in fetal alcohol syndrome. In: West, JR., editor. Alcohol and Brain Development. Oxford University Press; New York: 1986. p. 158-166.

Clarren SK, Alvord EC Jr, Sumi SM, Streissguth AP, Smith DW. Brain malformations related to prenatal exposure to alcohol. J Pediatrics 1978;92:64-67.

Coles CD, Platzman KA, Lynch ME, Freides D. Auditory and visual sustained attention in adolescents prenatally exposed to alcohol. Alcohol Clin Exp Res 2002;26:263-271. [PubMed: 11964567]

Coles CD, Platzman KA, Raskind-Hood CL, Brown RT, Falek A, Smith IE. A comparison of children affected by prenatal alcohol exposure and attention deficit, hyperactivity disorder. Alcohol Clin Exp Res 1997;21:150-161. [PubMed: 9046388]

Coulter CL, Leech RW, Schafer B, Scheithauer BW, Brumback RA. Midline cerebral dysgenesis, dysfunction of the hypothalamic-pituitary axis, and fetal alcohol effects. Arch Neurol 1993;50:771775. [PubMed: 8323485]

Devinsky, O.; D’Esposito, M. Neurology of Cognitive and Behavioral Disorders. Oxford University Press; New York: 2004.

Hamilton DA, Kodituwakku P, Sutherland RJ, Savage DD. Children with fetal alcohol syndrome impaired at place learning but not cued-navigation in virtual Morris water task. Beh Brain Res 2003;143:85-94.

Harwood, H. Report prepared by The Lewin Group for the National Institute on Alcohol Abuse and Alcoholism. 2000. Updating estimates of the economic costs of alcohol abuse in the United States: Estimates, update methods, and data.

Jacobson SW, Jacobson JL, Sokol RJ. Effects of fetal alcohol exposure on infant reaction time. Alcohol Clin Exp Res 1994;5:1125-1132. [PubMed: 7847594]

Jeret JS, Serur D, Wisniewski K, Fisch C. Frequency of agenesis of the corpus callosum in the developmentally disabled population as determined by computerized tomography. Pediatric Neuroscience 1986;12:101-103. [PubMed: 2428024]

Johnson VP, Swayze VW II, Sato Y, Andreasen NC. Fetal alcohol syndrome: Craniofacial and central nervous system manifestations. Am J Med Genetics 1996;61:329-339. [PubMed: 8834044]

Jones KL, Smith DW. Recognition of the fetal alcohol syndrome in early infancy. Lancet 1973;2:9991001. [PubMed: 4127281]

Jones KL, Smith DW. The fetal alcohol syndrome. Teratol 1975;12:1-10.

Jones KL, Smith DW, Ulleland CN, Streissguth AP. Pattern of malformation in offspring of chronic alcoholic mothers. Lancet 1973;1:1267-1271. [PubMed: 4126070]

Kim J, Thompson R. Cerebellar circuits and synaptic mechanisms involved in classical eyeblink conditioning. TINS 1997;20:177-181. [PubMed: 9106359]

Kodituwakku PW, Kalberg W, May PA. The effects of prenatal alcohol exposure on executive functioning. Alcohol Res Health 2001;25:192-198. [PubMed: 11810957]

Lee KT, Mattson SN, Riley EP. Classifying children with heavy prenatal alcohol exposure using measures of attention. J Int Neuropsychol Soc 2004;10:271-277. [PubMed: 15012847]

Ma X, Coles CD, Lynch ME, LaConte SM, Zurkiya DW, Hu X. Evaluation of corpus callosum anisotrophy in young adults with fetal alcohol syndrome using diffusion tensor imaging. Alcohol Clin Exp Res 2005;29:14-22.

Mattson SN, Goodman AM, Caine C, Delis DC, Riley EP. Executive functioning in children with heavy prenatal alcohol exposure. Alcohol Clin Exp Res 1999;23:1808-1815. [PubMed: 10591598] 
Mattson SN, Riley EP. A review of the neurobehavioral deficits in children with fetal alcohol syndrome or prenatal exposure to alcohol. Alcohol Clin Exp Res 1998;22:279-294. [PubMed: 9581631]

Mattson SN, Riley EP, Gramling L, Delis DC, Jones KL. Neuropsychological comparison of alcoholexposed children with or without physical features of fetal alcohol syndrome. Neuropsychology 1998;12:146-153. [PubMed: 9460742]

Mattson SN, Riley EP, Jernigan TL, Ehlers CL, Delis DC, Jones KL. Fetal alcohol syndrome: A case report of neuropsychological, MRI and EEG assessment of two children. Alcohol Clin Exp Res 1992;16:1001-1003. [PubMed: 1443415]

Mattson SN, Riley EP, Jernigan TL, Garcia A, Kaneko WM, Ehlers CL. A decrease in the size of the basal ganglia following prenatal alcohol exposure: A preliminary report. Neurotoxicol Teratol 1994;16:283-289. [PubMed: 7935262]

Mattson SN, Riley EP, Sowell ER, Jernigan TL, Sobel DF, Jones KL. A decrease in the size of the basal ganglia in children with fetal alcohol syndrome. Alcohol Clin Exp Res 1996;20:1088-1093. [PubMed: 8892532]

May PA, Gossage JP. Estimating the prevalence of fetal alcohol syndrome: A summary. Alcohol Res Health 2001;25:159-167. [PubMed: 11810953]

McClelland JL, Goddard NH. Considerations arising from a complementary learning systems perspective on hippocampus and neocortex. Hippocampus 1996;6:654-665. [PubMed: 9034852]

O'Hare ED, Kan E, Yoshii J, Mattson SN, Riley EP, Thompson PM, Toga AW, Sowell ER. Mapping cerebellar vermal morphology and cognitive correlates in prenatal alcohol exposure. NeuroReport 2005;16:1285-1290. [PubMed: 16056126]

Olson HC, Feldman JJ, Streissguth AP, Sampson PD, Bookstein FL. Neuropsychological deficits in adolescents with fetal alcohol syndrome: Clinical findings. Alcohol Clin Exp Res 1998;22:19982012. [PubMed: 9884144]

Peiffer J, Majewski F, Fishbach H, Bierich JR, Volk B. Alcohol embryo-and fetopathy. J Neuro Sci 1979;41:125-137.

Riikonen R, Salonen I, Partanen K, Verho S. Brain perfusion SPECT and MRI in foetal alcohol syndrome. Dev Med Child Neurol 1999;41:652-659. [PubMed: 10587040]

Riley EP, Mattson SN, Sowell ER, Jernigan TL, Sobel DF, Jones KL. Abnormalities of the corpus callosum in children prenatally exposed to alcohol. Alcohol Clin Exp Res 1995;19:1198-1202. [PubMed: 8561290]

Roebuck TM, Mattson SN, Riley EP. A review of the neuroanatomical findings in children with fetal alcohol syndrome or prenatal exposure to alcohol. Alcohol Clin Exp Res 1998a;22:339-344. [PubMed: 9581638]

Roebuck TM, Simmons RW, Mattson SN, Riley EP. Prenatal exposure to alcohol affects the ability to maintain postural balance. Alcohol Clin Exp Res 1998b;22:252-258. [PubMed: 9514315]

Roebuck-Spencer TM, Mattson SN, Marion SD, Brown WS, Riley EP. Bimanual coordination in alcoholexposed children: Role of the corpus callosum. J Int Neuropsychol Soc 2004;10:536-548. [PubMed: 15327732]

Sampson PD, Streissguth AP, Bookstein FL, Little RE, Clarren SK, Dehaene P, Hanson JW, Graham JM Jr. Incidence of fetal alcohol syndrome and prevalence of alcohol-related neurodevelopmental disorder. Teratol 1997;56:317-326.

Sowell ER, Jernigan TL, Mattson SN, Riley EP, Sobel DF, Jones KL. Abnormal development of the cerebellar vermis in children prenatally exposed to alcohol: size reduction in lobules I-V. Alcohol Clin Exp Res 1996;20:31-34. [PubMed: 8651458]

Sowell ER, Mattson SN, Thompson PM, Jernigan TL, Riley EP, Toga AW. Mapping callosal morphology and cognitive correlates: Effects of heavy prenatal alcohol exposure. Neurology 2001b;57:235-244. [PubMed: 11468307]

Sowell ER, Thompson PM, Mattson SN, Tessner KD, Jernigan TL, Riley EP. Voxel-based morphometric analyses of the brain in children and adolescents prenatally exposed to alcohol. Neuroreport 2001a; 12:515-523. [PubMed: 11234756]

Sowell ER, Thompson PM, Mattson SN, Tessner KD, Jernigan TL, Riley EP. Regional brain shape abnormalities persist into adolescence after heavy prenatal alcohol exposure. Cereb Cortex 2002a; 12:856-865. [PubMed: 12122034] 
Sowell ER, Thompson PM, Peterson BS, Mattson SN, Welcome SE, Henkenius AL. Mapping cortical gray matter asymmetry patterns in adolescents with heavy prenatal alcohol exposure. Neuroimage 2002b;17:1807-1819. [PubMed: 12498754]

Sowell ER, Thompson PM, Tessner KD, Toga AW. Mapping continued brain growth and cortical gray matter density reduction in frontal cortex: Inverse relationships during post adolescent brain maturation. J Neurosci 2001c;21:8819-8829. [PubMed: 11698594]

Streissguth AP, Bookstein FL, Sampson PD, Barr HM. Attention: prenatal alcohol and continuities of vigilance and attentional problems from 4 through 14 years. Dev Psychopathol 1995;7:419-446.

Streissguth AP, Sampson PD, Olson HC, Bookstein FL, Barr HM, Scott M, Feldman J, Mirsky AF. Maternal drinking during pregnancy: Attention and short-term memory in 14-year-old offspring--a longitudinal prospective study. Alcohol Clin Exp Res 1994;18:202-218. [PubMed: 8198221]

Swayze VW II, Johnson VP, Hanson JW, Piven J, Sato Y, Giedd JN. Magnetic resonance imaging of brain anomalies in fetal alcohol syndrome. Pediatrics 1997;99:232-240. [PubMed: 9024452]

Wisniewski K, Dambska M, Sher JH, Qasi Q. A clinical neuropathological study of the fetal alcohol syndrome. Neuropediatrics 1983;14:197-201. [PubMed: 6686290] 